\title{
Scale, key, and contour in the discrimination of tuned and mistuned approximations to melody
}

\author{
ANTHONY J. WATKINS \\ University of Reading, Reading, England
}

\begin{abstract}
Approximations to melody were synthesized using a computational technique whereby the occurrences of certain notes and intervals were made more probable than others. In experimental melodies, these notes and intervals were chosen to clarify the diatonic scale and the key of the melody. To clarify the scale of a melody, the probabilities of the diatonic scale notes were made higher than those of nonscale notes. Perception of these melodies was compared with control melodies in which the higher probabilities were assigned to a different set of notes. The latter were chosen so that no single diatonic scale embraced the set. To clarify the key of a melody, intervals with a low "fifth-span" were made more probable than intervals with a high fifth-span, while control melodies had the higher probabilities assigned to intervals with a high fifth-span. Here fifth-span is the separation of two notes measured along a progression of fifths. Listeners heard pairs of melodies and were asked to discriminate between pairs of different melodies and pairs in which the second melody was an exact transposition of the first. It was found that this discrimination was easier if the first melody contained a high proportion of diatonic-scale notes. A similar perceptual advantage held for melodies composed of intervals with low fifth-spans. Discrimination was also easier if the contour of the second melody in a different-pair differed from that of the first. The melodies were then mistuned by changing the size of the quantal interval (semitone) by a constant (log) factor. In this way, two types of mistuned melodies were generated: melodies for which the quantal interval was reduced, and melodies for which the quantal interval was increased. Discrimination advantages for diatonic scale and low fifth-span were not found for these mistuned melodies. Nevertheless, differences in contour still led to improved discrimination. It is concluded that melody perception draws on music-specific perceptual knowledge as well as more general factors. Mistuning destroys the perceptual advantages found for the melodies with a clear scale and a clear key. These attributes are therefore considered to be specific to music. By contrast, the perceptual salience of pitch contours is more general in origin, because it was shown for both the tuned and the mistuned tone sequences.
\end{abstract}

This paper is concerned with the effects that "mistuning" might have on the perceptual discrimination of melody pairs. For "tuned" melodies the note frequencies are based on the values of 12-tone equal temperament. This is a 12 -fold division of the $2: 1$ frequency ratio of the octave, forming logarithmically equal semitones. Since all other intervals are integer multiples of the semitone, it may be thought of as the "quantal interval." For "mistuned" melodies, this set of note frequencies is either expanded or compressed. This is done by increasing or decreasing the size of the quantal interval by a constant $(\log )$ amount. Physical discrepancies between melody pairs are therefore proportional to the size of the quantal

Dave Martin advised on the design and construction of the on-line facilities. Mary Dyson provided discussion and practical help. I am grateful to Mark Haggard, John Chalmers, and an anonymous reviewer for their comments on an earlier draft of the manuscript. These data were first reported at the 104th meeting of the Acoustical Society of America at Orlando, Florida, in November 1982.

The author's mailing address is: Department of Psychology, University of Reading, Reading RG6 2AL, England. interval. However, perceptual effects may depend on the relationships between notes and the way these influence perceptual organization. Tuning was varied to identify organizational factors that are specifically musical in origin, in that they depend on 12-tone equal-temperament tuning. These are distinguished from other factors that affect auditory processing more generally, in that they operate independently of tuning.

There have been some previous studies on the effects of distorting the absolute sizes of the intervals of a melody. Werner (1940) reduced the quantal interval to $16.5 \%$ of its usual size. He reported that for four of his five listeners the resulting melodies took on the perceptual characteristics of undistorted melodies (e.g., "melodious," "'major," "minor'). They were also able to recognize transpositions and produce appropriate transcriptions of these "micromelodies." However, this effect required a considerable period of training (up to 4 months) in which the listeners were slowly introduced to the new scale on a step-by-step basis. Shorter exposures to mistuned melodies lead to a dramatic impairment of melody identification. This effect was reported by White (1960), who 
doubled the quantal interval, by Idson and Massaro (1978), who halved the quantal interval, and by Moore and Rosen (1979), who used a compressed range of notefrequencies (quantal interval $=30 \%$ of its usual value) as well as an expanded range (quantal interval doubled). Idson and Massaro also showed that the perception of octave equivalence ("chroma") influences melody identification. They found that identification was improved if the mistuning preserved the original chroma. Moore and Rosen reported that listeners came to find the mistuned melodies "almost acceptable as music," and all three studies showed that despite the reduction in performance, the mistuned intervals did seem to be conveying some information. In the present study, three types of melodic information were considered: melodic contour, scale, and key. All three have been shown to influence melody discrimination, and the aim was to discover the extent of their influence on the learning and discrimination of both tuned and mistuned tone sequences.

Idson and Massaro (1978) concluded that much of the melodic information communicated by their mistuned melodies was the "contour." Contour refers to an "ordinal'" representation (S. S. Stevens, 1946) of the intervals of a melody. It shows whether adjacent notes are higher or lower than one another (Jones, 1976). Therefore, a melody such as "Three Blind Mice" would be represented as: down, down, up, down, down. Dowling and Fujitani (1971) have shown that transpositions of different novel melodies that share the same contour tend to sound like transpositions of the same melody. Other workers have shown that contour information can aid melody identification (Idson \& Massaro, 1978; Kallman \& Massaro, 1979; Massaro, Kallman, \& Kelly, 1980), although this is limited because different melodies often share the same contour. It has been suggested that various aspects of the contour are important for the communication of structure in music. For example, Jones (1981) proposed that reversals in contour might emphasize notes of structural importance, and it has been shown that contour reversals in novel melodies are more perceptually salient than are nonreversals (Dyson \& Watkins, 1984). Other authors maintain that contour is an important component in memory for music (Dowling, 1978). There is also much discussion of melodic contour in the terms of general perceptual principles, notably those of Gestalt psychology (e.g., Deutsch, 1978; Divenyi \& Hirsh, 1974, 1978; Heise \& Miller, 1951; Ortmann, 1926; Rosner \& Meyer, 1982), on the assumption that various visual and auditory contours are perceived in broadly similar ways. Indeed, listeners can accurately draw the contours of melodies they have just heard (Davies \& Jennings, 1977; Welker, 1982).

Melodies of western tonal music are commonly based on a highly constrained subset of the 12-fold division of the octave, and such subsets are known as scales (see Burns \& Ward, 1982). One pervasive example is the 7 note diatonic scale (see Helmholtz, 1885/1954). These notes are formed by dividing the octave into the following semitone steps:

$$
2,2,1,2,2,2,1 \text {. }
$$

Krumhansl (1979) and Krumhansl and Shepard (1979) have provided evidence for the perceptual distinctiveness of the diatonic notes. Krumhansl (1979) also showed that when melodies are based on diatonic notes, the initial note is better remembered than the initial note of nondiatonic melodies. Scale has been shown to influence melody discrimination by Dowling (1978). He found that exact transpositions of two different melodies were perceptually confused if they were based on a common scale (see Also Francès, 1958). Dewar, Cuddy, and Mewhort (1977) and Cuddy, Cohen, and Miller (1979) have also shown that, for musically trained listeners, melodies that obey the "musical rules of scale and tonal relationship" are more easily recognized than melodies that violate these rules.

In many tonal melodies of western origin there is one note that acts as a "tonal center" called the keynote. Bartlett and Dowling (1980) have shown that transpositions of two different melodies tend to be confused if their keynotes are closely related, although a similar effect reported by Cuddy et al. (1979) depended on the melodies and the differences between them. In these studies, key proximity was measured by the smallest separation of the keynotes measured around the circle of fifths. One way of representing this circle is shown in Figure 1.

If seven notes are adjacent on the circle of fifths they constitute a diatonic scale. The keynote and other scale steps are always in the same relative position within this set. For the diatonic major scale, the (clockwise) order

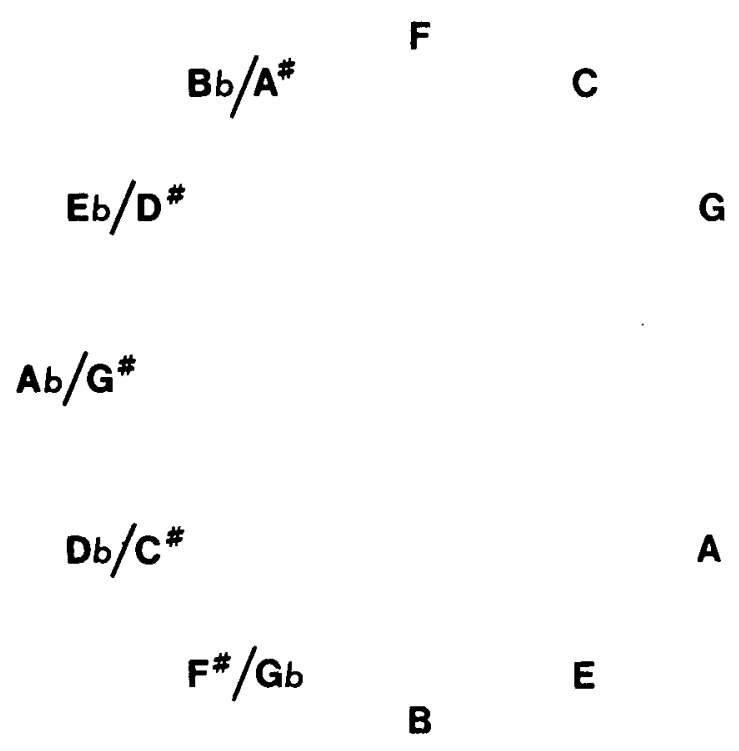

Figure 1. The circle of fifths. 
is: fourth, tonic, fifth, second, sixth, third, seventh (see Balzano, 1980, 1982).

Longuet-Higgins (1976-1978) has outlined certain constraints that key imposes on the succession of intervals in an unaccompanied melody. These concern the "fifthspan" of the intervals. This is the separation of two notes measured along a (noncircular) progression of fifths. In his analysis, the progression is restricted to the range of notes between 5 fifths below the keynote through 6 fifths above the keynote. The number of fifths from the keynote is represented by an integer, $q$. The fifth-span of an interval is the difference in $q$ between the two notes (absolute value). Figure 2 shows an example of this progression for the case in which the keynote is C. Accidentals are notated by choosing the appropriate flat for negative values of $q$, and the appropriate sharp for positive values of $q$.

The $q$ of notes in a two-octave range around the keynote are shown in Table 1 . The q of other notes may be found by a simple extrapolation. An important consequence of the restrictions on this fifth progression is that the fifth-span of intervals-other than the tritone-will depend on the choice of keynote. For example, the interval G-to-D is 1 fifth in some keys (such as F), but 11 fifths in $\mathrm{Db}$. Similarly, C-to-D is either 2 fifths or 10 fifths, depending on the key. C-to-A is 3 fifths or 9 fifths, and so on. Analysis of a large number of existing melodies showed that successive fifth-spans tended to be less than 6 fifths. Fifth-spans larger than this were rare. Any large fifth-spans that did occur were not found in adjacent intervals. These observations show that such constraints may provide listeners with information about the key of the melody: intervals (other than the tritone) will suggest a range of likely keynotes, for which the fifth-span of the interval is less than 6 . These intervals will also suggest a range of unlikely keynotes, for which the fifth-span of the interval is greater than 6. This type of information might be especially useful when other indicators of key (e.g., harmony) are ambiguous or absent. A computer program that operated with these constraints was able to establish the key of a range of existing melodies, given only information about the succession of note-frequencies. There was one additional constraint that this program needed to take into account. This concerned the interpretation of ascending semitones that end on notes between 2 through 5 fifths above the keynote (i.e., in the key of $\mathrm{C}$, the intervals: Db-to-D, Ab-to-A etc.). According to the above analysis, such an interval would be considered unlikely-having a fifth-span of 7-and might encourage

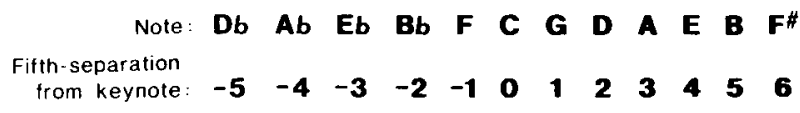

Figure 2. The progression of fifths and fifth separation from keynote (q) for the key of $C$.
Table 1

The Semitone Separation from Keynote and Corresponding Fifth Separation from Keynote for a 2-Octave Range

\begin{tabular}{ccc}
\hline \multicolumn{2}{c}{$\begin{array}{c}\text { Semitone Separation } \\
\text { from Keynote }\end{array}$} & $\begin{array}{c}\text { Fifth Separation } \\
\text { from } \\
\text { Keynote }\end{array}$ \\
\cline { 2 - 3 } Above & Below & 0 \\
0 & -12 & -5 \\
1 & -11 & 2 \\
2 & -10 & -3 \\
3 & -9 & 4 \\
4 & -8 & -1 \\
5 & -7 & 6 \\
6 & -6 & 1 \\
7 & -5 & -4 \\
8 & -4 & 3 \\
9 & -3 & -2 \\
10 & -2 & 5 \\
11 & -1 & 0 \\
12 & 0 & \\
\hline
\end{tabular}

the listener to perceive modulation to a key consistent with the smaller fifth-span, that is, 5. However, these intervals appear to be special cases, and fulfill an "ornamental" role that does not change the key. To accommodate this, the q of the initial note of this type of interval is taken as 5 fifths above the $q$ of the second note. This prompts a change in the notation of the accidentals so that the intervals (in the key of C) are now: C\#-to-D, G\#-to-A, etc. The fifth-span of the interval is now 5 , and so consistent with the original key.

Intervals with a fifth-span of 5 or less are traditionally called "diatonic" intervals, whereas those with a fifthspan greater than 5 are called "nondiatonic" (LonguetHiggins, 1976). Nevertheless, the above constraints do not restrict the melody to any particular diatonic scale, not even at a "local" level. This is because adjacent "diatonic" intervals (with a fifth-span less than 6) need not be compatible with the same diatonic scale. It is possible, in principle at least, for the above fifth-span constraints to indicate any of the 12 keynotes, regardless of the diatonic scale of the melody. However, in Western music, the keynote is also a member of the scale (Helmholtz, 1885/1954).

In this study, an adapted form of the recognition paradigm of Dowling and Fujitani (1971) was used. The listener must discriminate pairs of melodies that are exact transpositions of one another, from pairs of different melodies. (An exact transposition involves shifting the melody to a new starting note while holding constant the relative interval sizes.) The melodies used were initially novel. They were repeated over a series of trials to allow some learning to take place. The melodies are more properly described as approximations to melody: they were synthesized with the aim of incorporating a high proportion of notes and intervals likely in a "real" melody, using a nonexhaustive set of melodic constraints. Many factors are not considered, for example, rhythm. Nevertheless, the melodies are perceived as being similar in nature to 
"real" melodies (Watkins, 1981). Two factors are of current interest. One aims to clarify the scale of the melody by increasing the probability of diatonic-scale notes ("scale factor"). The other aims to clarify the key of the melody by increasing the probability of intervals with low fifth-spans ("fifth-span factor"). Both factors may be varied to generate control melodies: analogous principles are used, but in such a way that the resulting melodies do not have a clearly defined scale or key. These control melodies are perceived as being less similar to real melodies than their "melodic" counterparts (Watkins, 1981). The efficacy of a factor is determined by comparing performance on the experimental ("'melodic") form against that for the appropriate control. A third factor determines whether contour is changed within a "different" melody pair. Tuning is varied to investigate the extent to which the perceptual effects of these factors are specifically musical in origin. This also serves as a check on the adequacy of the controls: if the constraints employed to clarify scale and key are producing their effects by way of some nonmusical artifact (e.g., by generating contours that are easier to perceive, by biasing the occurrence of smaller intervals, by creating more redundant sequences, etc.), then they should not be disrupted by mistuning. Two experiments are reported. The first investigated whether the scale and contour factors influenced melody discrimination, and whether any observed influences were affected by mistuning. The second experiment investigated the influence of the fifth-span and contour factors while tuning was varied.

\section{METHODS}

Listeners were presented with pairs of melodies and asked to discriminate between pairs in which the second melody was an exact transposition of the first and pairs that contained different melodies. Each melody of the pair was 14 notes long. The transposition factor was selected randomly from trial to trial, being between three and five quantal intervals up or down. When novel melodies appear on each trial, this task is difficult, even for musically sophisticated listeners. Indeed, Dowling and Fujitani found this to be the case for their short five-note sequences. However, performance levels improve considerably if the first melody of the pair is held constant over a series of trials. In these experiments, 12 successive trials contained the same initial melody and immediate feedback was provided for the listener after his or her response to a pair of melodies.

The tone sequences used were synthetic approximations to melody, as described below. The scale and fifth-span factors vary the way in which the first melody of the pair is generated. The contour factor varies the way in which "different" melodies are generated. The tuning factor varies the frequency ratio of the quantal interval on which the melodies are based.

\footnotetext{
Melody Approximations

Sequences of 14 notes were produced as follows. The first note was selected at random from the range $\mathrm{C}=261.6 \mathrm{~Hz} \pm 5$ quantal intervals. This is designated the keynote. The remainder of the sequence was then synthesized note by note by selection from a 2 -octave range centered on the keynote. This selection combined a random process with probabilistic constraints, giving different probabilities of occurrence for the various notes. The final step was always forced to be a return to the keynote. At every other step,
}

the probability of occurrence of notes in the range was determined by both the note and the interval formed with the previous note. The probability of each note was determined by cross-multiplying three weightings. The values of the weightings were determined by independent constraints on the occurrence of notes and intervals. Different forms of these weightings were combined to produce different types of tone sequence in the two experiments.

One constraint was that the intervals formed were more likely to be less than 6 semitones. The relevant weightings are shown in Table 2.

A rationale for this "old golden rule of composition" is discussed by Divenyi and Hirsh (1978), who also present evidence that tone sequences containing smaller intervals are easier to organize perceptually than are sequences of larger intervals (see also Deutsch, 1978). Two other constraints operate at the same time, and govern the fifth-span of the intervals and the occurrence of scale notes. They operate in one of two ways, depending on whether the melody is intended to represent a melodic form of the scale or fifth-span factor or to be a control.

\section{Scale Factor, Diatonic or Nondiatonic}

The weightings used for the different forms of the scale factor are shown in Table 3.

When the scale factor is in its melodic (diatonic) form, the seven notes of the diatonic major scale are made more probable. In the notation of Longuet-Higgins these are notes with $q$ in the range of -1 through +5 . The control form of this factor (nondiatonic) emphasizes notes obtained from the following semitone divisions of the octave:

$$
2,2,1,2,1,2,1,1 \text {. }
$$

These eight notes were reduced to seven by excluding the keynote. This control is therefore comparable with the melodic form, being based on a fairly even division of the octave. The crucial difference is that there is no one diatonic scale that encompasses these notes. This is because the notes are well scattered over the cycle of fifths (Balzano, 1980, 1982), with $q=-4,-2,-1,1,2,4$, and 5 .

\section{Fifth-Span Factor, Low or High}

To clarify the key of a melody, the constraints outlined by Longuet-Higgins $(1976,1978)$ were used. These constraints refer to the intervals of a melody described by their separation along a progression of fifths in the range from 5 fifths below the keynote through 6 fifths above the keynote (Figure 2). The fifth-span of an interval may be calculated by finding the number of fifths from the keynote for each of its notes, then subtracting these values and taking the absolute value of the result.

Table 2

Weightings that Increase the Probability of Smaller Semitone Steps

\begin{tabular}{|c|c|c|}
\hline \multicolumn{2}{|c|}{ Semitone Span of Interval } & \multirow{2}{*}{$\begin{array}{c}\text { Relative } \\
\text { Weighting }\end{array}$} \\
\hline Ascending & Descending & \\
\hline 1 & -1 & 8 \\
\hline 2 & -2 & 8 \\
\hline 3 & $-\overline{3}$ & 7 \\
\hline 4 & -4 & 6 \\
\hline 5 & -5 & 6 \\
\hline 6 & -6 & 2 \\
\hline 7 & -7 & 2 \\
\hline 8 & -8 & 2 \\
\hline 9 & -9 & 2 \\
\hline 10 & -10 & 2 \\
\hline 11 & -11 & 2 \\
\hline \multicolumn{2}{|c|}{ All Others } & 0 \\
\hline
\end{tabular}


Table 3

Weightings Used for the Scale Factor to Increase the Probability of Occurrence of Notes of a Diatonic Scale (Column 2) or a Nondiatonic Set of Notes (Column 3)

\begin{tabular}{ccc}
\hline $\begin{array}{c}\text { Fifth-Separation } \\
\text { from Keynote }\end{array}$ & \multicolumn{2}{c}{ Relative Weighting } \\
\cline { 2 - 3 } & Diatonic & Nondiatonic \\
\hline-5 & 9 & 9 \\
-4 & 9 & 64 \\
-3 & 16 & 16 \\
-2 & 16 & 121 \\
-1 & 121 & 121 \\
0 & 256 & 9 \\
1 & 169 & 121 \\
2 & 121 & 256 \\
3 & 121 & 9 \\
4 & 64 & 169 \\
5 & 64 & 64 \\
6 & 9 & 16 \\
\hline
\end{tabular}

The weightings used for the different forms of the fifth-span factor are shown in Table 4.

The melodic form of the factor (low fifth-span), makes intervals having a fifth-span less than 6 more likely than others. For the control form (high fifth-span), intervals with a fifth-span between 6 and 11 are more probable. It is also necessary to use two rules. For the melodic form of the factor, the first rule is that intervals with a fifth-span greater than 5 must not occur in adjacent intervals. The second rule concerns the assignment of $q$ in certain ascending semitones. If the second note of such an interval is $2,3,4$, or 5 fifths from the keynote, then the first note of the interval would ordinarily be $-5,-4,-3$, or -2 fifths from the keynote, respectively, giving the interval a fifth-span of 7 . In other contexts, such a large fifth-span encourages the perception of modulation, but in this case it does not. Therefore, in this context only, the $q$ of the first note of the interval is changed to $7,8,9$, or 10 fifths from the keynote, respectively, giving the interval a fifth-span of 5 . The control forms of these rules constrain the sequence to a similar extent, but in an inappropriate way. This was done by assigning incorrect values of $q$ to each note for the purpose of applying the rules. These incorrect values were a random rearrangement of the correct values. There was no correlation between correct and control values of $q$ across notes.

\section{Combining Constraints}

To determine the probability of any one note, all three constraints must be considered. Two of the constraints govern interval sizes, so the previous note will affect the probability of the next. For each note of the melody, the probability of each next note is calculated. This is done by cross-multiplying three numbers. These are the weighting of the interval determined by semitone-span (Table 2), the weighting of the note determined by scale (Table 3 ), and the weighting of the interval determined by fifth-span (Table 4). This is done for all possible next notes, and the resulting distribution is normalized and scaled so that its sum is unity. A random-number selector then picks the next note with the appropriate biases. This note is appended to the sequence unless it violates the rules associated with the fifth-span factor, or if it is simply a repetition of the previous note. Such failures require the random-number selector to try again. In this way, numerous examples of four different types of melody can be synthesized. In Experiment 1, the effect of scale was examined by comparing diatonic with nondiatonic melodies, where both have low fifth-span. In Experiment 2, the effect of fifthspan was examined by comparing low with high fifth-span melodies, both types being diatonic.

\section{Contour Factor, Same or Different}

When it is required that the second melody of a pair be different from the first, the following procedure is adopted. A region of permissible variation around each note of the first melody is defined. In the simplest case, this has boundaries that are four quantal intervals above and below the note. To keep both melodies within a 2-octave range around the initial note, boundaries that lie outside this range are suitably coniracted. Also, if the note of the first melody is above the keynote, then the boundaries for the second-melody note must also be above the keynote, and vice versa. Boundaries are therefore contracted to comply with this constraint. A new melody is then synthesized by selecting notes at random from each of these regions in turn. At each step, it is necessary to decide whether it is possible to change the contour of the original. If it is, then the regions are further restricted so that the contour of the original is either preserved or violated, depending on the value of the contour factor, that is, contour same or contour different. For the former, a final check ensures that the resulting melody is different from the first, and for the latter a check is made to make sure that there are some contour differences. The only other restrictions are that the initial and final notes remain the same, that is, as the keynote, and that there be no successive repetitions. The second melody is then transposed as described above.

\section{Tuning Factor, Quantal Interval $=\mathbf{6 0}$, 100 , or 140 cents}

In equal-temperament tuning there is a constant frequency ratio relating the notes of adjacent semitones. It is therefore possible to define an integer for each note that represents the number of semitone steps (or quantal intervals) from some reference note frequency (see Hahn \& Jones, 1981). If $\mathrm{f}_{\mathrm{o}}$ is the frequency of the reference note and $\mathrm{p}$ is the number of quantal intervals from this reference, then:

$$
\mathrm{f} / \mathrm{f}_{\mathrm{o}}=2^{\mathrm{p} / 12},
$$

where $f$ is the frequency of the note in hertz. To vary the frequency ratio of the quantal interval by a constant amount, a scaling factor, $c$, is introduced:

$$
\mathrm{f} / \mathrm{f}_{\mathrm{o}}=2^{\mathrm{cp} / 12},
$$

where $\mathrm{c}=1.0$ for the usual 12-tone equal-temperament notes, that

Table 4

Weightings Used for the Fifth-Span Factor, which Increase the Probability of Occurrence of Intervals with Low (Melodic) or High (Control) Fifth-Span

\begin{tabular}{cccc}
\hline \multirow{2}{*}{$\begin{array}{c}\text { Fifth-Span } \\
\text { of } \\
\text { Interval }\end{array}$} & $\begin{array}{c}\text { Low } \\
\text { Fifth-Span } \\
\text { (Experiment 1) }\end{array}$ & $\begin{array}{c}\text { Low } \\
\text { Fifth-Span } \\
\text { (Experiment 2) }\end{array}$ & $\begin{array}{c}\text { High } \\
\text { Fifth-Span } \\
\text { (Experiment 2) }\end{array}$ \\
\hline 0 & 6 & 216 & 64 \\
1 & 8 & 512 & 8 \\
2 & 8 & 512 & 8 \\
3 & 7 & 343 & 27 \\
4 & 6 & 216 & 64 \\
5 & 6 & 216 & 64 \\
6 & 2 & 8 & 512 \\
7 & 2 & 8 & 512 \\
8 & 2 & 8 & 512 \\
9 & 2 & 8 & 512 \\
10 & 2 & 8 & 512 \\
11 & 2 & 8 & 512 \\
\hline
\end{tabular}


is, quantal interval $=1$ semitone. If $c<1.0$, then the quantal interval is contracted. If $c>1.0$, then the quantal interval is expanded. The number of equal-temperament cents in the quantal interval is c multiplied by 100 . The value of the reference note used here is $f_{o}=261.6 \mathrm{~Hz}$ ("middle C").

The tuning factor has three values, where the quantal interval is 60,100 , or 140 cents. The tuning factor is implemented after the melody approximations have been computed. It has no effect on the method of computation.

\section{Designs}

In Experiment 1, the fifth-span factor was held constant in a melodic (low) form and scale, contour, and tuning were varied. Each of three groups of 20 listeners was given one type of tuning. The listeners learned melodies differing in scale (diatonic or nöndiatonic), with both types of contour for the "different" melodies (same or different). Experiment 2 was similar, except that the scale factor was kept constant in its melodic form (diatonic) and fifthspan was either low or high. In addition, the fifth-span factor was emphasized in Experiment 2. This was done by increasing the difference between the probability weightings for more and less probable fifth-spans. The rationale here came from pilot work on the form of the fifth-span factor necessary for any discrimination advantages.

\section{Apparatus and Procedure}

Listeners were sampled from a group of undergraduate students. Some were educated in music, but generally not beyond secondaryschool level. They did not describe themselves as musically sophisticated. None reported any hearing problems.

The listeners were run individually, allowing different randomizations of melodies, melody orders, and trial orders to be used for each of them. Each listener's session was 12 blocks of 12 trials with a short rest between blocks. Twelve different melodies were synthesized for each session, one for each block. Either the scale factor (Experiment 1) or the fifth-span factor (Experiment 2) was varied between blocks, with the melodic and control forms of each factor taking six blocks per session. The total number of "first melodies" synthesized for Experiment 1 was therefore 2 (diatonic vs. nondiatonic) $\times 6$ (examples per session) $\times 3$ (tunings) $\times 20$ (listeners) $=720$ melodies. For Experiment 2, where fifth-span was varied, an additional 720 melodies were synthesized.

Trial type was "same," "different with contour same," or "different with contour different." Each had an equal probability of occurrence on any one trial. The initial note of the first melody and the transposition of the second were selected randomly from the stated ranges on a trial by trial basis. The second melody of "different" pairs was similarly randomized.

Each trial consisted of a pair of 14-note melodies separated by a 2-sec gap. After each pair of melodies, the listeners were given as long as they wished to indicate whether the melodies differed, and whether they were sure or not. This was done by selecting one of four buttons. An oscilloscope display then gave them appropriate feedback before the next pair of melodies was presented. Nonparametric assessments of the areas under ROCs were calculated for each listener, giving $\mathrm{p}(\mathrm{A})$ values (see $\mathrm{McNicol}, 1972$ ). These values run from 0.5 , at chance performance, up to a score of 1.0 meaning perfect discrimination. An arcsin transform of these values preceded further data reduction.

The melodies were played at 4 notes/sec, with each note lasting for its full $1 / 4 \mathrm{sec}$. Onsets and offsets were at zero-crossings of the waveform. The notes were produced by varying the period of a digital square wave, followed by a 10-bit digital-to-analogue conversion at $35,971 \mathrm{samples} / \mathrm{sec}$. Note periods were accurate to the nearest integer number of sample periods, and these were within $0.4 \%$ of 12 -tone equal-temperament values. The signals were lowpass filtered at $2 \mathrm{kHz}$, with a cutoff slope of $24 \mathrm{~dB}$ per octave. They were presented to the listener diotically via Sennheiser HD-424 earphones in the quiet of an IAC-1201 booth. The level was attenuated to roughly $30 \mathrm{~dB}$ above the threshold of one audiometrically nor- mal listener. System hum and noise were inaudible. A computing system based on a PDP-8 synthesized the melodies, administered the experiment, and analyzed the data.

\section{RESULTS}

\section{Experiment 1}

Discrimination scores were well above the chance level for all combinations of factor values but below the performance ceiling for all listeners. Analysis of variance of the transformed $p(A)$ values showed two significant main effects and one significant first-order interaction, all with $\mathrm{p}<.001$. There were no other significant $\mathrm{F}$ ratios. The main effect of the contour factor, with $\mathrm{F}(1,171)=$ 41.64 , is illustrated in Figure 3. Here the means are pooled across diatonic and nondiatonic melodies and expressed as a function of tuning. Each data point is based on 120 different melodies, or 1,440 trials. There was no interaction with the contour factor. Therefore, this result is a simple superiority for contour-different trials over

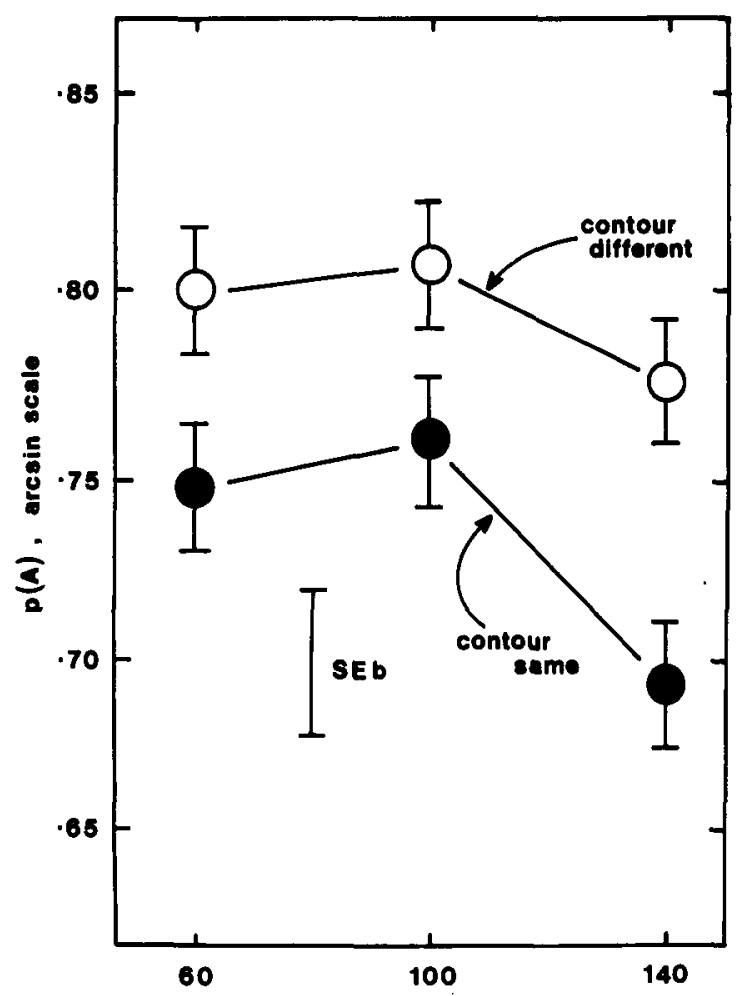

Cente per quantal interval

Figure 3. Results of Experiment 1. The discrimination index, $p(A)$, plotted against the three values of the tuning factor. Values shown are the means pooled across listeners and across the diatonic and nondiatonic values of the scale factor. Open circles $=$ different contour, closed circles = same contour. Bars around the means are standard errors of differences appropriate for comparing means within the same level of the tuning factor (i.e., within groups). The bar labeled SEb is the standard error of differences appropriate for comparisons between levels of the tuning factor (i.e., between groups). 
contour-same trials that does not vary with the tuning or the scale of the melodies. The other significant $F$ ratios were a main effect of scale, with $F(1,171)=22.56$, and a scale by tuning interaction, where $\mathrm{F}(2,171)=7.91$. These are illustrated in Figure 4, where means are pooled across contour-same and contour-different trials. These data show that the melodies based on a diatonic scale are easier to learn and discriminate than their controls, but only when the melodies are correctly tuned to the values of 12-tone equal temperament (i.e., 100 cents per quantal interval). This superiority is not found when the melodies are mistuned; here the effects of the scale factor are negligible.

\section{Experiment 2}

Again, performance was well above chance but below ceiling. Analysis of variance showed just four significant F ratios: three main effects and one first-order interaction. There were main effects for contour, where $F(1,171)=$ 42.80 with $p<.001$, and for tuning, where $F(2,57)=$ 6.27 with $\mathrm{p}<.01$. These are illustrated in Figure 5, where means are pooled across low and high fifth-span and expressed as a function of tuning. Contour is the parameter. There were no interactions with the contour factor. Therefore, the contour effect is a simple superiority for contourdifferent over contour-same trials, which is not influenced by the fifth-span or tuning of the melodies. There is a main

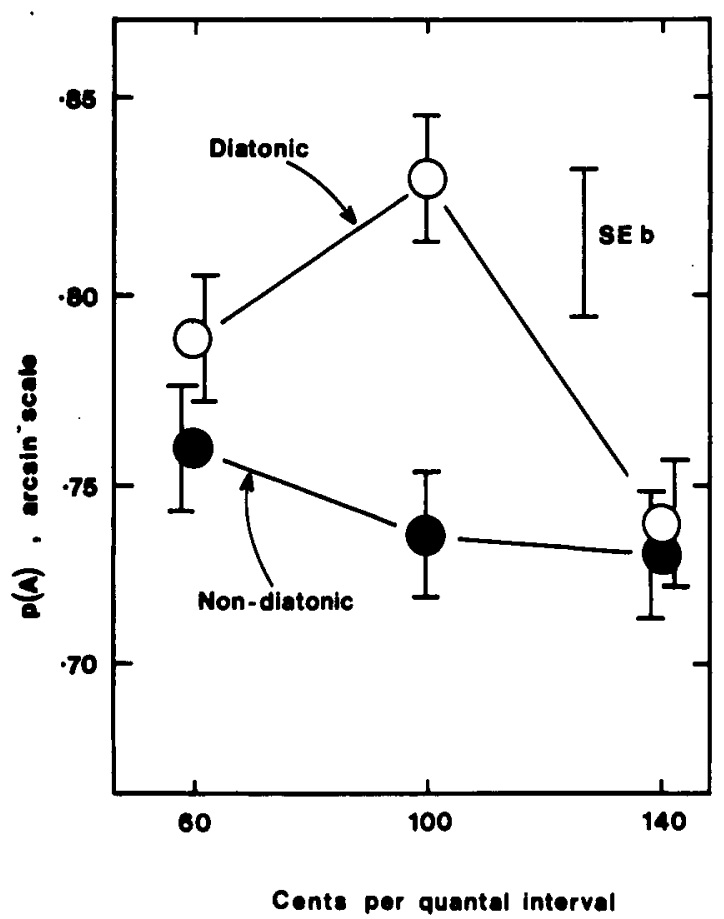

Figure 4. Results of Experiment 1. Means are pooled across contour-same and contour-different trials. Open circles $=$ diatonic scale, closed circles = nondiatonic scale. Within- and between-group standard errors of differences are represented in the same way as in Figure 3.

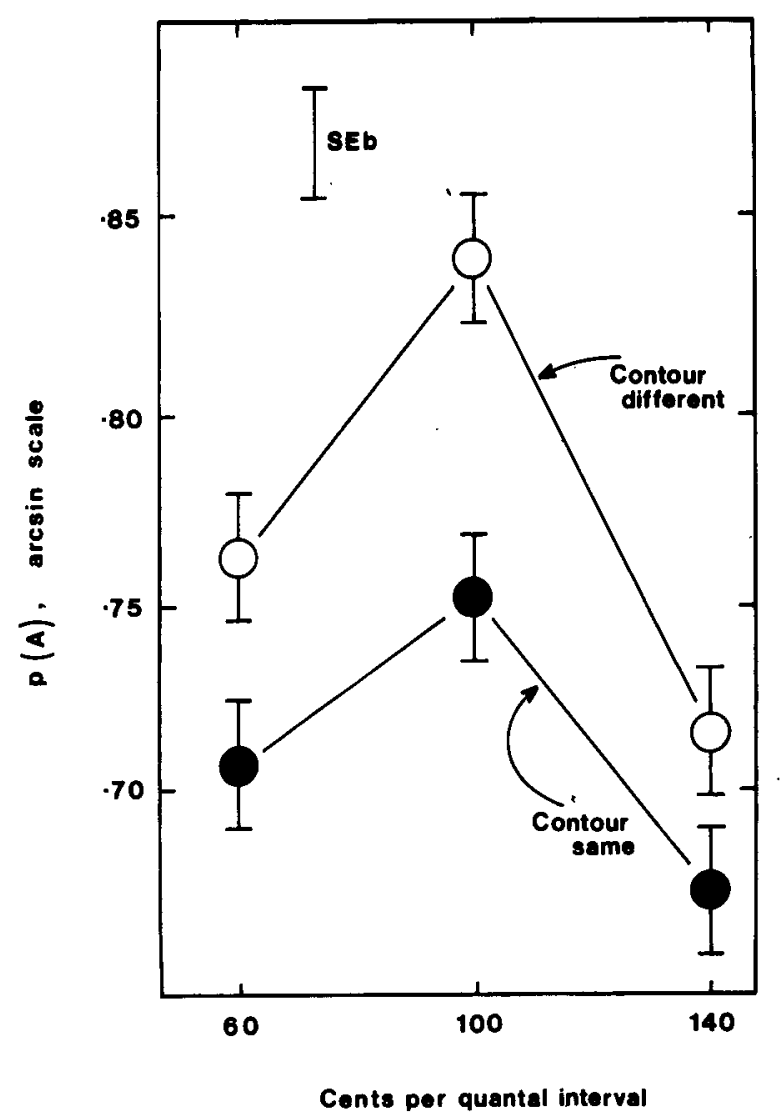

Figure 5. Results of Experiment 2 showing the effects of the tuning and contour factors. Open circles = contour different, closed circles $=$ contour same. Means are pooled across low and high fifthspan. See the caption of Figure 3 for other details.

effect of tuning in this experiment, and this appears as a superiority for "correct" equal-temperament tuning over both types of "mistuning." However, the tuning effect is a little more subtle than this because of an interaction between tuning and fifth-span, where $F(2,171)=$ 6.80 with $p<.01$. There is also a main effect of fifth-span, where $F(1,171)=12.61$ with $p<.001$. Figure 6 plots means pooled across contour-same and contour-different trials as a function of tuning. This shows superior discrimination for melodies based on a low fifth-span over controls. This is found only for melodies tuned to the values of 12-tone equal temperament. Mistuning destroys this superiority, as shown.

\section{DISCUSSION}

In the experiments described, it was found that scale, key (fifth-span), and contour affect melody discrimination. Contour differences between a pair of melodies aid the discrimination of correctly tuned melodies. They also aid the discrimination of mistuned melodies in which the 


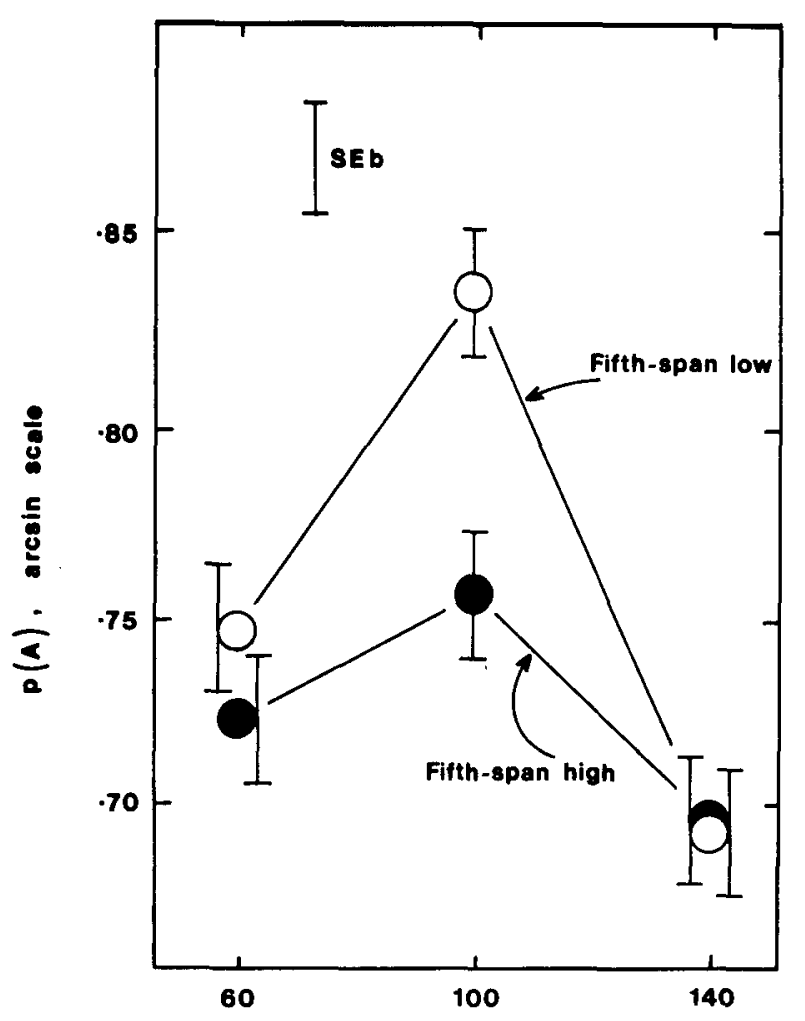

Cents per quantal interval

Figure 6. Results of Experiment 2 showing the effects of the fifthspan and tuning factors. Open circles = low fifth-span, closed circles = high fifth-span. Within- and between-group standard errors are as previously shown.

quantal interval is expanded or contracted. There are discrimination advantages for diatonic over nondiatonic melodies and for low fifth-span over high fifth-span melodies, but these effects were found only for 12-tone equaltemperament tuning. Therefore, these experiments show that the discrimination of complex tone sequences is not governed solely by the "physical" discrepancies between them, that is, as measured by differences in note frequencies. The effects of mistuning appear to depend on more subtle interactions with factors concerning perceptual organization.

There is an apparent inconsistency between the results of the two experiments. Experiment 2 shows an overall superiority for 12-tone equal-temperament tuning, but Experiment 1 does not. The Experiment 2 result may be explained by considering the way in which the melodies are generated. That is, all melodies were synthesized with the scale factor in its melodic form, so the Experiment 1 results would lead us to expect mistuning to impair perception of all melodies; hence the overall tuning effect. On the other hand, all Experiment 1 melodies were syn- thesized with low fifth-spans, so why is there no overall effect of mistuning here? The most probable answer is that the form of the fifth-span factor differs between experiments, being emphasized in Experiment 2, as stated previously. Pilot work had shown that the form of the fifth-span factor used in Experiment 1 has only a small effect on recognition memory, and hence the lack of an overall tuning effect in this experiment. One other possibility is that there is some interaction between the scale and fifth-span factors such that fifth-span is effective if the scale factor is melodic, but not vice versa.

Why is it that listeners can "adapt" to mistuned contours, but seem unable to extract scale and key information from these severely mistuned melodies? One possibility is that mistuning destroys the relationships between the frequency ratios of intervals and the harmonics of note frequencies. Here, this mismatch is highlighted because the melodies were played with harmonically rich square waves. This relationship is the basis of "simple ratio" hypotheses about the origins of scales. The idea is that the emergence of the scale notes was influenced by psychoacoustical aspects of consonance, and that tuning systems evolved to maximize sets of notes related by small whole-number frequency ratios. (e.g., Plomp, 1976; Terhardt, 1977, 1978). It is possible to restore the appropriate relationship between intervals and harmonics while preserving the mistuning. This has been done by using notes where the partials are appropriately "stretched" or "condensed" (Cohen, 1979; Mathews \& Pierce, 1980; McAdams, 1982; Slaymaker, 1970). These studies show that appropriate stretching of both the tuning and the partials can restore the perception of consonance for chords. However, the "musical" attributes of chords (e.g., "major," "'minor") and sequences of chords (e.g., the sense of "finality" associated with cadences) are not perceived. Pierce (1983) attributes this result to the fact that pitch perception is governed by the harmonic relationship of partials (Schouten, Ritsma, \& Cardozo, 1962) and prevents perceptual adaptation to this "new music."

Alternative reasons for the lack of flexibility of scale and key perception derive from the hypotheses underlying various "microtonal pitch systems." Several authors have considered tuning systems based on quantal intervals smaller than a semitone (Balzano, 1980, 1982; Chalmers, 1979; Glasier, 1978; Partch, 1974; Rothenberg, 1978a, 1978b; Schafer \& Piehl, 1947; von Hoerner, 1974, 1976; Yunik \& Swift, 1980). These authors do not envisage that the new scale should be a simple "condensed" version of the correctly tuned scale notes: different sets of notes are predicted to emerge' in each case, and these depend on the degree of note-frequency compression. These hypotheses lead one to expect that a mistuned melody will be perceived as originating from a more finely divided octave. Therefore, the scale and key of the original should be destroyed. It is therefore possible that there is more flexibility in scale and key perception than is apparent here. This might be shown with different note frequen- 
cies for the mistuned scales, or with different sounds. However, there is no perceptual evidence in favor of these speculations.

Other ideas about the origin of scales are inherently more adaptable; for example, the view that scales derive from a need to reduce the information content of melodies to within psychologically acceptable limits (Dowling, 1978; Krumhansl \& Shepard, 1979) or that they are learned through contact with the pervasive musical idiom (Deutsch \& Feroe, 1981). However, the present results show that any potential for a rapid adaptation to differently tuned scales has been lost by the present group of listeners. Indeed, Deutsch and Feroe (1981) stress the robust nature of their "alphabets" (scales) by describing them as "overlearned." Also, in a developmental study, Krumhansl and Keil (1982) found that the perceptual distinctiveness of the diatonic notes was present at an early age. Nevertheless, Werner's (1940) results do suggest that some relearning is possible, given an extensive period of training.

The introspections of the present group of listeners were comparable to those reported by Moore and Rosen (1979); that is, they rapidly found that the novel tunings "didn't seem too bad really," and, indeed, some found that they lost all awareness of any mistuning. Such reports are not too surprising, since all tunings led to high performance levels, so that listeners were able to remember, transpose, and compare the tone sequences. It is difficult to believe that listeners can do this if the tone sequences are devoid of meaning. However, the present experiments show that this ability does not require the perception of scale and key, and is therefore unlikely to be a unique property of melody perception. Indeed, there are other sounds for which such abilities are useful, notably in the perception of the fundamental frequency contours of speech. Here, contour similarities need to be recognized in various frequency ranges ("transpositions") and across varying degrees of frequency excursion ("tunings"). It has been argued that speech contours and melodic contours have similar perceptual properties (Dyson \& Watkins, 1984; Thomassen, 1982). This argument is reinforced by the present findings, that the discrimination of contour differences in both melodic and nonmelodic tone sequences is quantitatively similar, there being no reliable differences in the size of the contour effect across the different tunings. It may be concluded that contour discrimination is generally similar for a variety of sounds. This may be because rudimentary organizational principles govern the perception of all contours (e.g., Gestalt laws), but might also be explained by listeners' drawing on perceptual strategies normally used in perceiving the pitch of the voice.

One caveat should be noted. This is that the design of these and other discrimination experiments does not allow us to decide whether some contours are more easily remembered than others. Nor does it illuminate possible interactions between scale, key, and contour type. This is also true of identification experiments, to the extent that contour is merely used in discriminating between possible answers. Therefore, the idea that contour is used in the communication of musical structure (Jones, 1981; Rosner \& Meyer, 1982) needs to be investigated in other ways, especially since contour discrimination does not seem to be uniquely melodic. A more appropriate test of these views would require the construction of sequences with structurally "good" contours, and a comparison of the learning of these with appropriate control melodiesthat is, an approach similar to that adopted for the fifthspan and scale factors in the present study.

The lack of flexibility associated with the perception of scale and key is apparent for the perception of other complex sounds, notably those of speech. Sounds that might plausibly have been produced by a human vocal apparatus are perceived differently from sounds of comparable complexity that are not perceived as speech (e.g., Best, Morrongiello, \& Robson, 1981). There is also a learning superiority for speech over other complex sounds (House, K. N. Stevens, Sandel, \& Arnold, 1962). Some authors (e.g., Liberman, 1982; Repp, 1982) argue that this is evidence for a mode of perception for speech that exhibits unique properties. However, the present findings for melody are in many ways analogous to the speech perception results of House et al. Therefore, this general class of phenomena might be observable for other complex sounds that have environmental significance. Indeed, there have been many demonstrations that perceptual organization is aided by the similarity of environmental events to the "schemata" of familiar objects (see Attneave, 1981). This viewpoint provides a convenient interpretive framework for the present findings. The scale and fifthspan factors embody certain aspects of melody "schemata," and therefore generate melodies that are easier to learn and discriminate. The "fit" to melody schemata is destroyed by severe mistuning and so the perceptual advantage is no longer observed. This is consistent with the idea that a variety of specializations are part and parcel of perceptual organization and that the efficiency of "perceptual problem solving"' is bought at the cost of specificity and inflexibility (see Rock, 1975).

\section{CONCLUSION}

The present experiments show that there are two independent kinds of influence on the perception of melodies. On the one hand, there are factors that interact with the listener's knowledge of the pervasive musical idiom. Two of these are the scale and key of the melody as communicated by the scale and fifth-span constraints of these melody approximations. Listeners appear to be able to take advantage of these constraints to build a more accurate perceptual representation of the melodic melodies, possibly in the manner suggested by Attneave (1981). This results in better learning of such melodoies and less confusion with similar melodies presented soon afterwards. However, these factors are influential only if the tones are correctly tuned to the values of 12-tone equal tem- 
perament. If the tones are severely mistuned, the perceptual system is unable to adapt its problem-solving strategies appropriately, even though the problem is similar when expressed in terms of a physical description of the sounds. A similar lack of adaptability would also explain the poor performance for correctly tuned control melodies.

Other factors involved in the discrimination task are more general. This is illustrated here by the detection of similarities and differences in the contours of transposed melodies. Listeners are able to perceive these differences regardless of the tuning of the tones. This result contrasts sharply with the effects found for the scale and fifth-span factors. The reason may be that listeners are "importing" organizational strategies developed for the perception of the pitch contours of speech or other sounds. It is also possible that perceptual similarities between the latter and melodic contour have a common origin in some more rudimentary organizational principle.

\section{REFERENCES}

ATtNenve, F. (1981). Three approaches to perceptual organization. In M. Kubovy \& J. R. Pomerantz (Eds.), Perceptual organization. Hillsdale, NJ: Erlbaum.

Balzano, G. J. (1980). The group-theoretic description of 12-fold and microtonal pitch systems. Computer Music Journal, 4, 66-84.

BALzANo, G. J. (1982). The pitch set as a level of description for studying musical pitch perception. In M. Clynes (Ed.), Music mind and brain. New York: Plenum.

Bartlett, J. C., \& Dowling, W. J. (1980), Recognition of transposed melodies: A key-distance effect in developmental perspective. Journal of Experimental Psychology: Human Perception and Performance, 6, 501-515.

Best, C. T., Morrongiello, B., Robson, R. (1981). Perceptual equivalence of acoustic cues in speech and nonspeech perception. $\mathrm{Per}$ ception \& Psychophysics, 29, 191-211.

BURNS, E. M., WARD, D. W. (1982). Intervals scales and tuning. In D. Deutsch (Ed.), The psychology of music. London: Academic Press.

Chalmers, J. (1979). A collection of scales with nineteen tones. Xenharmonikon, Whole Nos. 7 and 8.

COHEN, E. (1979). Fusion and consonance relations for tones with inharmonic partials. Joumal of the Acoustical Society of America, 65, S123.

Cuddy, L. L., Cohen, A. J., Miller, J. (1979). Melody recognition: The experimental application of musical rules. Canadian Journal of Psychology, 33, 148-156.

DAvIES, J. B., \& JENNINGs, J. S. (1977). Reproduction of familiar melodies and the perception of tonal sequences. Joumal of the Acoustical Society of America, 61, 534-541.

Deutsch, D. (1978). The psychology of music. In E. C. Carterette \& M. P. Friedman (Eds.), Handbook of perception. New York: Academic Press.

Deutsch, D., Feroe, J. (1981). The internal representation of pitch sequences in tonal music. Psychological Review, 88, 503-522.

Dewar, K. M., Cuddy, L. L., \& MEwhort, D. J. K. (1977). Recognition memory for single tones with and without context. Journal of Experimental Psychology: Human Leaming and Memory, 3, 60-67.

DivenYi, P. L., HiRSH, I. J. (1974). Identification of temporal order in three-tone sequences. Journal of the Acoustical Society of America, 56, 144-151.

DivenYI, P. L., Hirsh, I. J. (1978). Some figural properties of auditory patterns. Journal of the Acoustical Society of America, 64, 1369-1385.
DowLING, W. J. (1978). Scale and contour: Two components of a theory of memory for melodies. Psychological Review, 85, 341-345.

Dowling, W. J., \& FuJTtani, D. S. (1971). Contour, interval and pitch recognition in memory for melodies. Journal of the Acoustical Society of America, 49, 524-531.

Dyson, M. C., WAtkins, A. J. (1984). A figural approach to the role of melodic contour in melody recognition. Perception \& Psychophysics, 35, 477-488.

Francès, R. (1958). La perception de la musique. Paris: Librarie Philosophique, J. Vrin.

Glasier, E. (1978). Confessions of a microtonal convert. Interval, 1, 2-3.

HAHN, J. JoNes, M. R. (1981). Invariants in auditory frequency relations. Scandinavian Journal of Psychology, 22, 129-144.

Heise, G. A., \& MiLler, G. A. (1951). An experimental study of auditory patterns. American Journal of Psychology, 64, 68-77.

Helmholtz, H. L. F. (1954). On the sensations of tone (A. J. Ellis, Trans.) New York: Dover. (Original work published 1885)

House, K. S., Stevens, K. N., SANDel, T. T., \& ARNOld, B. (1962). On the learning of speechlike vocabularies. Journal of Verbal Learning and Verbal Behavior, 1, 133-143.

IDSON, W. L., \& MASSARo, D. W. (1978), A bidimensional model of pitch in the recognition of melodies. Perception \& Psychophysics, 24, 551-556.

JONES, M. R. (1976). Levels of structure in the reconstruction of temporal and spatial serial patterns. Journal of Experimental Psychology: Human Learning and Memory, 2, 475-488.

JoNEs, M. R. (1981). A tutorial on some issues and methods in serial pattern research. Perception \& Psychophysics, 30, 492-504.

Kallman, H. J., \& Massaro, D. W. (1979). Tone chroma is functional in melody recognition. Perception \& Psychophysics, 26, 32-36.

KRUMHANSL, C. L. (1979). The psychological representation of musical pitch in a tonal context. Cognitive Psychology, 11, 346-374.

Krumhansl, C. L., KeIL, F. C. (1982). Acquisition of the hierarchy of tonal functions in music. Memory \& Cognition, 10, 243-251.

Krumhansi, C. L., Shepard, R. N. (1979). Quantification of the hierarchy of tonal functions within a diatonic context. Journal of $E x-$ perimental Psychology: Human Perception and Performance, 5, 579-594.

Liberman, A. M. (1982). On the finding that speech is special. American Psychologist, 37, 148-167.

Longuet-Higgins, H. C. (1976). Perception of melodies. Nature, 263, 646-653.

LoNGUET-Higgins, H. C. (1978). The perception of music. Interdisciplinary Science Reviews, 3, 148-156.

Massaro, D. W., Kallman, H. J., \& Kelly, J. L. (1980). The role of tone height, melodic contour, and tone chroma in melody recognition. Journal of Experimental Psychology: Human Leaming and Memory, 6, 77-90.

Mathews, M. V., \& Pierce, J. R., (1980). Harmony and nonharmonic partials. Joumal of the Acoustical Society of America, 68, 1252-1257.

MCADAMS, S. (1982). Spectral fusion and the creation of auditory images. In M. Clynes (Ed.), Music mind and brain. New York: Plenum.

MCNicol, D. (1972). A primer of signal detection theory. London: Allen and Unwin.

MOORE, B. C. J., \& Rosen, S. M. (1979). Tune recognition with reduced pitch and interval information. Quarterly Journal of Experimental Psychology, 31, 229-240.

Ortmann, O. (1926). On the melodic relativity of tones. Psychological Monographs, 35, 1-47.

PARTCh, H. (1974). Genesis of music. New York: Da Capo.

PierCe, J. R. (1983). The science of musical sound. New York: Freeman. PLOMP, R. (1976). Aspects of tone sensation. New York: Academic Press. REPP, B. H. (1982). Phonetic trading relations and context effects: New experimental evidence for a speech mode of perception. Psychological Bulletin, 92, 81-110.

Rock, I. (1975). An introduction to perception. New York: Macmillan. RosNer, B. S., \& MEYER, L. B. (1982). Melodic processes and the perception of music. In D. Deutsch (Ed.), The psychology of music. New York: Academic Press. 
RothenBERG, D. (1978a). A model for pattern perception with musical applications. Part I: Pitch structures as order preserving maps. Mathematical Systems Theory, 11, 199-234.

RoTHENBERG, D. (1978b). A model for pattern perception with musical applications. Part II: The information content of pitch structures. Mathematical Systems Theory, 11, 353-372.

Schafer, T., \& PIEHL, W. (1947). Musical instruments in nineteentone equal temperament. Journal of the Acoustical Society of America, 19, 750. (Abstract)

Schouten, J. F., Ritsma, R. J., \& Cardozo, B. L. (1962). Pitch of the residue. Journal of the Acoustical Society of America, 34, 1418-1424.

SlaymaKer, F. H. (1970). Chords from tones having stretched partials. Journal of the Acoustical Society of America, 47, 1569-1571.

STEVENS, S. S. (1946). On the theory of scales of measurement. Science, $103,677-680$.

TERHARDT, E. (1977). The two-component theory of musical consonance. in E. F. Evans \& J. P. Wilson (Eds.), Psychophysics and physiology of hearing. New York: Academic Press.

TERHARDT, E. (1978). Psychoacoustical evaluation of musical sounds. Perception \& Psychophysics, 23, 483-492.

Thomassen, J. M. (1982). Melodic accent: Experiments and a tenta- tive model. Journal of the Acoustical Society of America, 71, 1596-1605.

von Hoerner, S. (1974). Universal music? Psychology of Music, 2 , 18-28.

voN HoERnER, S. (1976). The definition of major scales, for chromatic scales of 12, 19 and 31 divisions per octave. Psychology of Music, 4, 12-23.

WATKINs, A. J. (1981). Tonal sequences, melody and music. In Proceedings of the Institute of Acoustics, Spring Conference. (pp. 347-350). Edinburgh: Institute of Acoustics.

WELKER, R. L. (1982). Abstraction of themes from melodic variations. Journal of Experimental Psychology: Human Perception and Performance, 8, 435-447.

WERNER, H. (1940). Musical 'micro-scales' and 'micro-melodies'. Journal of Psychology, 10, 149-156.

WHITE, B. W. (1960). Recognition of distorted melodies. American Journal of Psychology, 73, 100-107.

YuNIK, M. \& SwIFT, G. W. (1980). Tempered musical scales for sound synthesis. Computer Music Journal, 4, 60-65.

(Manuscript received September 5, 1984; revision accepted for publication February 10, 1985.) 